\title{
Omicron variant of SARS-CoV-2 harbors a unique insertion mutation of putative viral or human genomic origin
}

\author{
A.J. Venkatakrishnan ${ }^{1}$, Praveen Anand ${ }^{2}$, Patrick J. Lenehan ${ }^{1}$, Rohit Suratekar², \\ Bharathwaj Raghunathan ${ }^{3}$, Michiel J.M. Niesen ${ }^{1}$, Venky Soundararajan ${ }^{1,2,3 *}$ \\ ${ }^{1}$ nference, Cambridge, Massachusetts 02139, USA \\ 2 nference labs, Bengaluru, Karnataka 560017, India \\ ${ }^{3}$ nference, Toronto, ON M5V 1M1, Canada \\ *Correspondence to: Venky Soundararajan (venky@nference.net)
}

\begin{abstract}
The emergence of a heavily mutated SARS-CoV-2 variant (B.1.1.529, Omicron) and it's spread to 6 continents within a week of initial discovery has set off a global public health alarm. Characterizing the mutational profile of Omicron is necessary to interpret its shared or distinctive clinical phenotypes with other SARS-CoV-2 variants. We compared the mutations of Omicron with prior variants of concern (Alpha, Beta, Gamma, Delta), variants of interest (Lambda, Mu, Eta, lota and Kappa), and all 1523 SARS-CoV-2 lineages constituting 5.4 million SARS-CoV-2 genomes. Omicron's Spike protein has 26 amino acid mutations (23 substitutions, two deletions and one insertion) that are distinct compared to other variants of concern. Whereas the substitution and deletion mutations have appeared in previous SARS-CoV-2 lineages, the insertion mutation (ins214EPE) has not been previously observed in any SARS-CoV-2 lineage other than Omicron. The nucleotide sequence encoding for ins214EPE could have been acquired by template switching involving the genomes of other viruses that infect the same host cells as SARS-CoV-2 or the human transcriptome of host cells infected with SARS-CoV-2. For instance, given recent clinical reports of co-infections in COVID-19 patients with seasonal coronaviruses (e.g. HCoV-229E), single cell RNA-sequencing data showing co-expression of the SARSCoV-2 and HCoV-229E entry receptors (ACE2 and ANPEP) in respiratory and gastrointestinal cells, and HCoV genomes harboring sequences homologous to the nucleotide sequence that encodes ins214EPE, it is plausible that the Omicron insertion could have evolved in a co-infected individual. There is a need to understand the function of the Omicron insertion and whether human host cells are being exploited by SARS-CoV2 as an 'evolutionary sandbox' for host-virus and inter-viral genomic interplay.
\end{abstract}

\section{Introduction}

A new SARS-CoV-2 variant with an extensively mutated Spike protein was first reported to the World Health Organization (WHO) from South Africa on November 24, 2021, with the first sample collected on November 9, 2021. This strain has since been denoted as the Omicron variant (WHO nomenclature) and B.1.1.529 (PANGO lineage) ${ }^{1}$. The rapid assessment of the variant by The Technical Advisory Group on SARS-CoV-2 Virus Evolution and classification of Omicron as a variant of concern by the WHO within 48 hours has facilitated timely epidemiological surveillance. Since the initial discovery of Omicron, the variant has already been detected in over 20 countries across six continents. ${ }^{2,3}$ 
Thoroughly characterizing the mutational profile of Omicron is the necessary first step to begin interpreting its shared or distinctive clinical phenotypes, sensitivity or resistance to existing vaccines, and whether Omicron-like variants that evolve in the future may have heightened virulence. Indeed, SARS-CoV-2 has evolved into different variants of concern and variants of interest through a combination of missense, deletion, insertion, and other mutations. In the Spike (S) protein that engages the ACE2 receptor on human cells to facilitate viral entry, missense mutations (e.g. E484K) have led to significant changes in the Spike-ACE2 binding affinity, and deletions (e.g. $\Delta Y 144$ ) have modulated the effect of neutralizing anti-Spike antibodies ${ }^{4-9}$ Insertion mutations have been less prevalent in SARS-CoV-2 evolution. ${ }^{10}$ However, one of the most functionally consequential mutations in the evolutionary history of SARS-CoV-2 till date was the "PRRA" Spike protein insertion in the S1/S2 cleavage site, which introduced the polybasic FURIN cleavage site that mimics the RRARSVAS peptide in human ENAC-alpha. ${ }^{11-14}$ The availability of 5.4 million SARS-CoV-2 genomes covering 1523 lineages from over 200 countries/territories in the GISAID database from the beginning of the pandemic gives an opportunity to characterize the mutational profile of the Omicron variant in comparison to other SARS-CoV-2 variants.

In this study, we compare the mutational profile of Omicron with 1523 SARS-CoV-2 lineages including the variants of concern and variants of interest. We highlight that Omicron's Spike protein harbors an insertion mutation ins214EPE that is absent in all other SARS-CoV-2 lineages. Given the salience of viral genetic recombination and the debated plausibility of host genome integration by SARS-CoV-2 ${ }^{15-17}$, we considered a variety of host-viral and inter-viral genomic matter exchange scenarios that may have contributed to the adoption of this insertion mutation in the precursor variant of Omicron. We discuss potential sources for the origin of the ins214EPE and highlight the need to experimentally characterize the role of ins214EPE for transmission and immune evasion.

\section{Results and Discussion}

Comparison of mutations in Omicron to previous SARS-CoV-2 lineages shows the presence of a unique insertion mutation in Omicron's N-terminal domain (NTD)

The Omicron variant harbors 37 mutations in the Spike protein, which include six deletion mutations, one insertion mutation and 30 substitution mutations. ${ }^{18} 16$ of the 37 mutations are surge-associated mutations ${ }^{19}$ (Table 1), i.e. their mutational prevalence increased during any three-month window when COVID-19 cases surged. Comparing these Spike protein mutations in Omicron with pre-existing variants of concern (Alpha, Beta, Gamma and Delta) shows that 26 mutations are distinct to Omicron and 7 mutations overlap between Omicron and Alpha (Figure 1). We analyzed whether any of the 26 Omicron mutations appeared in the prior variants of interest (Lambda, Mu, Eta, lota and Kappa) or prior SARS-CoV-2 lineages by comparing with mutations from 5,382,852 genomes corresponding to 1523 lineages from the GISAID database (Table 1; Figure S1). Interestingly, the insertion mutation ins214EPE (Figure S2) has not been previously observed in any SARS-CoV-2 lineage other than Omicron, whereas the substitution and deletions mutations have appeared in previous SARS-CoV-2 lineages (Table S1). 
The EPE insertion on Omicron maps to the N-terminal domain (NTD) distal from the antibody binding supersite. ${ }^{9}$ However, the loop where the insertion is present maps to a known human T-cell epitope on SARS-CoV-2. ${ }^{20}$ Further studies will be necessary to understand whether this insertion may help SARS-CoV-2 escape T-cell immunity. ${ }^{10}$ Given the importance of the PRRA insertion giving rise to a polybasic FURIN cleavage site in the original SARS-CoV-2 strain, it is important to understand the functional significance and evolutionary origins of the ins214EPE insertion in the Omicron variant. ${ }^{11-14}$

\section{Origin of insEPE in Omicron potentially due to template switching using genome of co-infecting viruses or host}

The mutational burden of Omicron is higher in the Spike protein than the rest of the proteome (Figure S3). This highly mutated Spike variant harbors a novel insertion mutation ins214EPE. Although the position 214 appears to be an insertion hotspot ${ }^{10}$ the EPE insertion in Omicron appears to be novel. Previous analyses of sequences deposited in GISAID suggested that insertions in the SARS-CoV-2 genome likely arise from polymerase slippage or template switching. ${ }^{10,21}$ Template switching is a normal event during RNA synthesis for coronaviruses, as this process is used to generate sub-genomic RNAs (sgRNAs). ${ }^{22,23}$ In this process, also known as copy-choice recombination, the RNA-directed RNA polymerase (RdRp) and the nascent strand dissociate from the template RNA strand and reassociate with a new template (or the same template at a different position), and then RNA synthesis continues. Typically, such recombination involves templates with high sequence similarity ("homologous recombination"), although nonhomologous (or "illegitimate") recombination between dissimilar sequences can also occur. ${ }^{24}$

Recombination between SARS-CoV-2 lineages in the context of simultaneous co-infection has been observed, with particularly high recombination rates seen in the Spike protein sequence. ${ }^{22,25}$ The ins214EPE could have been acquired by template switching involving the genomes of SARS-CoV-2, other viruses that infect the same host cells as SARS-CoV-2, or the human transcriptome of host cells infected with SARS-CoV-2. Indeed, there have been clinical reports of COVID-19 patients also being infected with seasonal coronaviruses such as HCoV$229 \mathrm{E}^{26}$. Searching the HCoV-229E genome for homology to a nucleotide sequence encoding ins214EPE shows the presence of an identical sequence in HCoV-229E's Spike protein, which could have been exploited for template switching (Figure 2). Furthermore, based on analysis of single cell RNA seq data (Table S2), we see that the receptors of SARS-CoV-2 (ACE2) and HCoV-229E (ANPEP) are co-expressed in gastrointestinal (e.g. enterocytes) and respiratory tissues (e.g. respiratory ciliated cells). This gives rise to the plausibility of such cells in co-infected individuals being exploited as sites of genomic interplay between different viruses. In addition to co-infection with different coronaviruses, there have been reports of co-infection with SARS-CoV2 and other respiratory pathogens including non-SARS-CoV-2 Coronaviridae. ${ }^{27,28}$

It has been suggested previously that insertion mutations in the SARS-CoV-2 genomes could have originated from the human host genome ${ }^{29}$. Indeed, numerous fragments of the human genome and transcriptome harbor nucleotide sequences that are identical to the coding sequence of ins214EPE. There are over 750 fragments of the human genome with nucleotide sequences identical to the coding sequence of ins214EPE, which include mRNAs of SLCA7 and TMEM245 
as top hits (Figure S4). Of these, the transcripts that are expressed specifically in human host cells (e.g. alveolar cells, enterocytes) ${ }^{30}$ that are infected by SARS-CoV-2 could be candidates for the origin of the ins214EPE sequence. Thus, the evolution of the unique insertion in Omicron could have been based on template switching during viral co-infections, or from prevalent templates in the human genome.

Even as the production of COVID-19 vaccines is being scaled up, vaccine inequity and vaccine hesitancy have been speculated as contributors to the emergence of Omicron ${ }^{31,32}$. Since achieving global vaccination could take years, it is important to vigilantly monitor the changing mutational landscape that could lead to the emergence of new SARS-CoV-2 variants. Indeed, even among the Omicron variants there are differences in the prevalence of the constituent mutations (Figure S1). Finally, there is a need to sequence SARS-CoV-2 genomes from individuals with viral co-infections and in general to develop a "variant warning system" for early detection of variants of concern based on their mutational profile.

\section{Methods}

\section{Analysis of surge-associated mutations using GISAID database and OWID}

The core SARS-CoV-2 mutations associated with each of the parental strains: Alpha (B.1.1.7), Beta (B.1.351), Gamma (P.1), Delta (B.1.617.2), Lambda (C.37), Mu (B.1.621), Eta (B.1.525), lota (B.1.526) and Kappa (B.1.617.1) were derived from CoV-RDB database ${ }^{18}$. All the SARS-CoV-2 genome sequences corresponding to the Omicron variants along with the mutations were directly derived from GISAID database ${ }^{2,3}$ (121 SARS-CoV-2 genomes annotated as B.1.1.529 as on 29th November 2021). Analyzing the occurrences of ins214EPE in the GISAID database, besides their prevalence in the Omicron variants two occurrences are in genomes assigned to lineages B.1.1 and B.1.1.263, which is likely due to incorrect assignment of lineages. 10 other occurrences outside Omicron are in sequences that were yet to be assigned a lineage.

To understand which SARS-CoV-2 mutations correlates with COVID-19 test positivity, we extracted "Surge-Associated mutations" from GISAID (5,382,852 sequences from 202 countries/territories between December 2019 to November 2021) and Our World in Data (OWID) data $^{33}$ as described in previous study ${ }^{19}$. A surge associated mutation satisfies the following criteria: (1) it is present in at least 100 GISAID sequences; (2) it is present in a time window during which there is at least a $5 \%$ increase in test positivity, over three consecutive months (in a given country); (3) it is present in time window during which there is at least a $5 \%$ increase in sequence deposition with the given mutation over three consecutive months (in a given country).

\section{NCBI BLAST search to identify homologs to nucleotide sequence encoding Omicron's ins214EPE}

Aligning the Spike protein nucleotide sequences from Omicron and the reference Wuhan SARS-CoV-2 sequences shows that two different nucleotide sequence insertions can give rise to the sequencing encoding Omicron's ins214EPE. These insertion candidates are GAGCCAGAA and GCCAGAAGA. A NCBI BLAST search was performed for 'CGTGAGCCAGAAGAT' which 
includes both the possible insertion candidates (GAGCCAGAA, GCCAGAAGA), using default BLAST parameters (word size: 7; match/mismatch: -1/3; gap costs existence/extension: 5/2). The search against the database of human genome plus transcriptome database (Human G+T) containing 160592 sequences resulted in 763 hits. The search against human coronaviruses HCoV-OC43 (taxid:31631), HCoV-229E (taxid:11137), HCoV-NL63 (taxid:277944), HCoV-229E (taxid:11137) — resulted in 709 hits.

\section{Declaration of Interests}

AJV, PA, PJL, RS, MJMN and VS are employees of nference and have financial interests in the company. nference collaborates with bio-pharmaceutical companies on data science initiatives unrelated to this study. These collaborations had no role in study design, data collection and analysis, decision to publish, or preparation of the manuscript.

\section{References}

1. Classification of Omicron (B.1.1.529): SARS-CoV-2 Variant of Concern. https://www.who.int/news/item/26-112021-classification-of-omicron-(b.1.1.529)-sars-cov-2-variant-of-concern.

2. Shu, Y. \& McCauley, J. GISAID: Global initiative on sharing all influenza data - from vision to reality. Euro Surveill. 22, (2017).

3. GISAID - hCov19 Variants. https://www.gisaid.org/hcov19-variants/.

4. Wang, P. et al. Antibody resistance of SARS-CoV-2 variants B.1.351 and B.1.1.7. Nature (2021) doi:10.1038/s41586-021-03398-2.

5. Uriu, K. et al. Neutralization of the SARS-CoV-2 Mu Variant by Convalescent and Vaccine Serum. N. Engl. J. Med. (2021) doi:10.1056/NEJMc2114706.

6. Collier, D. A. et al. Sensitivity of SARS-CoV-2 B.1.1.7 to mRNA vaccine-elicited antibodies. Nature 593, 136141 (2021).

7. McCarthy, K. R. et al. Recurrent deletions in the SARS-CoV-2 spike glycoprotein drive antibody escape. Science 371, 1139-1142 (2021).

8. Motozono, C. et al. SARS-CoV-2 spike L452R variant evades cellular immunity and increases infectivity. Cell Host Microbe 29, (2021).

9. McCallum, M. et al. N-terminal domain antigenic mapping reveals a site of vulnerability for SARS-CoV-2. Cell 184, 2332-2347.e16 (2021).

10. Garushyants, S. K., Rogozin, I. B. \& Koonin, E. V. Template switching and duplications in SARS-CoV-2 genomes give rise to insertion variants that merit monitoring. Communications Biology 4, 1-9 (2021).

11. Anand, P., Puranik, A., Aravamudan, M., Venkatakrishnan, A. J. \& Soundararajan, V. SARS-CoV-2 strategically mimics proteolytic activation of human ENaC. Elife 9, (2020).

12. Coutard, B. et al. The spike glycoprotein of the new coronavirus 2019-nCoV contains a furin-like cleavage site absent in CoV of the same clade. Antiviral Res. 176, (2020).

13. Jaimes, J. A., Millet, J. K. \& Whittaker, G. R. Proteolytic Cleavage of the SARS-CoV-2 Spike Protein and the Role of the Novel S1/S2 Site. iScience 23, (2020).

14. Structure, Function, and Antigenicity of the SARS-CoV-2 Spike Glycoprotein. Cell 181, 281-292.e6 (2020).

15. Zhang, L. et al. Reverse-transcribed SARS-CoV-2 RNA can integrate into the genome of cultured human cells and can be expressed in patient-derived tissues. Proc. Natl. Acad. Sci. U. S. A. 118, (2021).

16. Parry, R., Gifford, R. J., Lytras, S., Ray, S. C. \& Coin, L. J. M. No evidence of SARS-CoV-2 reverse transcription and integration as the origin of chimeric transcripts in patient tissues. Proceedings of the National Academy of Sciences of the United States of America vol. 118 (2021).

17. Zhang, L. et al. Response to Parry et al.: Strong evidence for genomic integration of SARS-CoV-2 sequences 
and expression in patient tissues. Proceedings of the National Academy of Sciences of the United States of America vol. 118 (2021).

18. Tzou, P. L. et al. Coronavirus Antiviral Research Database (CoV-RDB): An Online Database Designed to Facilitate Comparisons between Candidate Anti-Coronavirus Compounds. Viruses 12, (2020).

19. Venkatakrishnan, A. J. et al. Antigenic minimalism of SARS-CoV-2 is linked to surges in COVID-19 community transmission and vaccine breakthrough infections. medRxiv 2021.05.23.21257668 (2021).

20. Tarke, A. et al. Comprehensive analysis of T cell immunodominance and immunoprevalence of SARS-CoV-2 epitopes in COVID-19 cases. Cell Rep Med 2, 100204 (2021).

21. Chrisman, B. S. et al. Indels in SARS-CoV-2 occur at template-switching hotspots. BioData Min. 14, (2021).

22. Jackson, B. et al. Generation and transmission of interlineage recombinants in the SARS-CoV-2 pandemic. Cell 184, 5179-5188.e8 (2021).

23. Sawicki, S. G. \& Sawicki, D. L. Coronaviruses use discontinuous extension for synthesis of subgenome-length negative strands. Adv. Exp. Med. Biol. 380, (1995).

24. Simon-Loriere, E. \& Holmes, E. C. Why do RNA viruses recombine? Nat. Rev. Microbiol. 9, $617-626$ (2011).

25. Turkahia, Y. et al. Pandemic-Scale Phylogenomics Reveals Elevated Recombination Rates in the SARS-CoV-2 Spike Region. bioRxiv 2021.08.04.455157 (2021) doi:10.1101/2021.08.04.455157.

26. Lau, S. K. P. et al. Molecular Evolution of Human Coronavirus $229 E$ in Hong Kong and a Fatal COVID-19 Case Involving Coinfection with a Novel Human Coronavirus 229E Genogroup. mSphere 6, (2021).

27. Musuuza, J. S. et al. Prevalence and outcomes of co-infection and superinfection with SARS-CoV-2 and other pathogens: A systematic review and meta-analysis. PLoS One 16, e0251170 (2021).

28. Kim, D., Quinn, J., Pinsky, B., Shah, N. H. \& Brown, I. Rates of Co-infection Between SARS-CoV-2 and Other Respiratory Pathogens. JAMA 323, 2085-2086 (2020).

29. thomasppeacock. Putative host origins of RNA insertions in SARS-CoV-2 genomes. https://virological.org/t/putative-host-origins-of-rna-insertions-in-sars-cov-2-genomes/761 (2021).

30. Venkatakrishnan, A. J. et al. Knowledge synthesis of 100 million biomedical documents augments the deep expression profiling of coronavirus receptors. Elife 9, (2020).

31. Wroughton, L. Officials: Variants 'haunt' world with vaccine imbalance between rich and poor nations. The Washington Post (2021).

32. Head, M. Omicron Is Here: A Lack of COVID Vaccines Is Partly Why. https://www.scientificamerican.com/article/omicron-is-here-a-lack-of-covid-vaccines-is-partly-why1/.

33. Mathieu, E. et al. A global database of COVID-19 vaccinations. Nat Hum Behav 5, 947-953 (2021).

34. Doddahonnaiah, D. et al. A Literature-Derived Knowledge Graph Augments the Interpretation of Single Cell RNA-seq Datasets. Genes 12, (2021).

35. Martin, J. C. et al. Single-Cell Analysis of Crohn's Disease Lesions Identifies a Pathogenic Cellular Module Associated with Resistance to Anti-TNF Therapy. Cell 178, (2019).

36. Chua, R. L. et al. COVID-19 severity correlates with airway epithelium-immune cell interactions identified by single-cell analysis. Nat. Biotechnol. 38, (2020). 


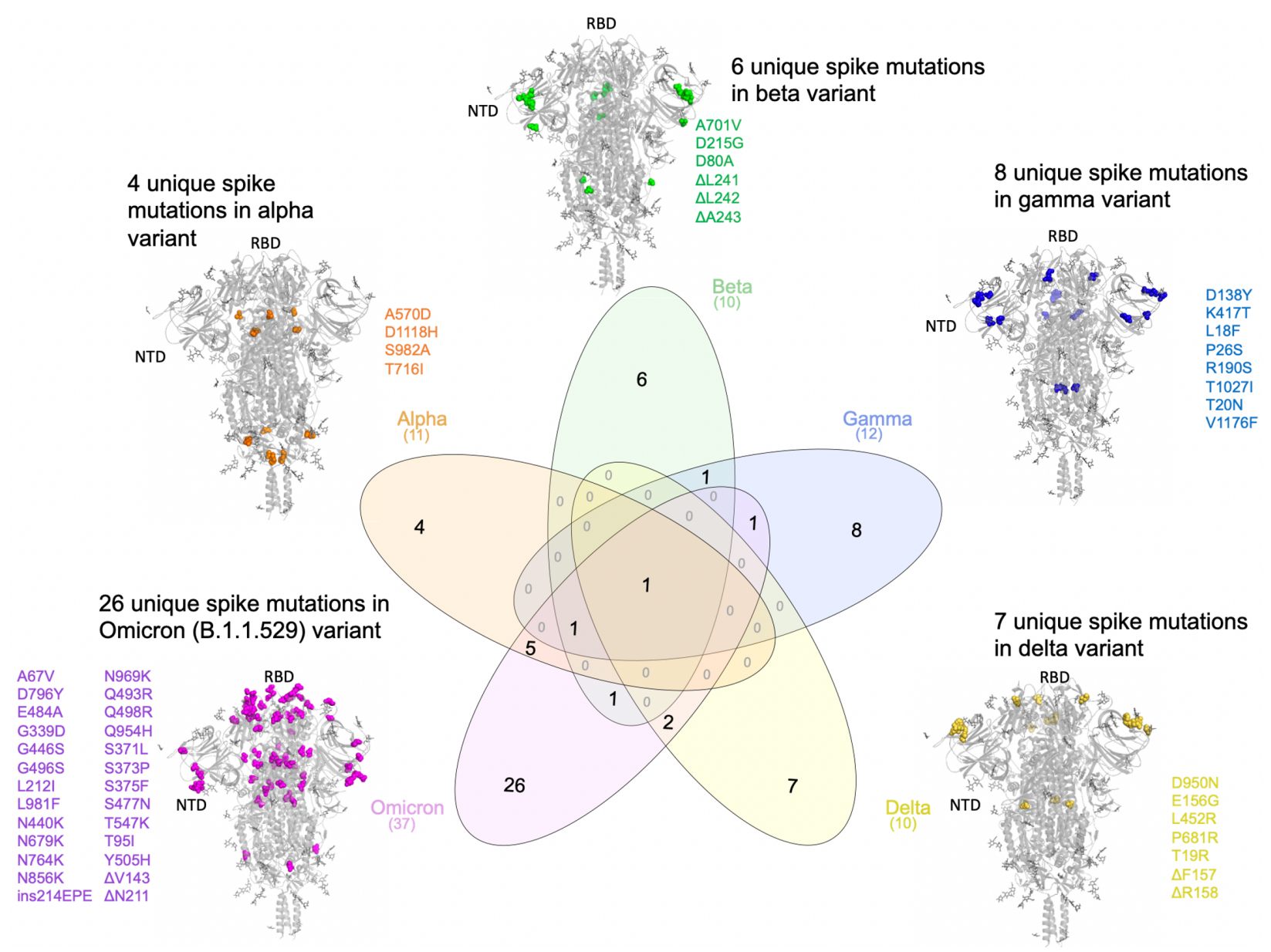

Figure 1. Venn diagram depicting the overlap of lineage specific spike mutations in the SARS-CoV2 variants of concern. The unique key mutations observed in the spike protein for each of the variants are highlighted (spheres) on the homo-trimeric Spike protein of SARS-CoV-2. The B.1.1.529 (Omicron) variant has the highest number (26) of unique mutations in the spike protein from this perspective, making its emergence a "step function" in evolution of SARS-CoV-2 strains. 
a. Potential mechanism of template switching leading to the generation of the ins214EPE in Omicron

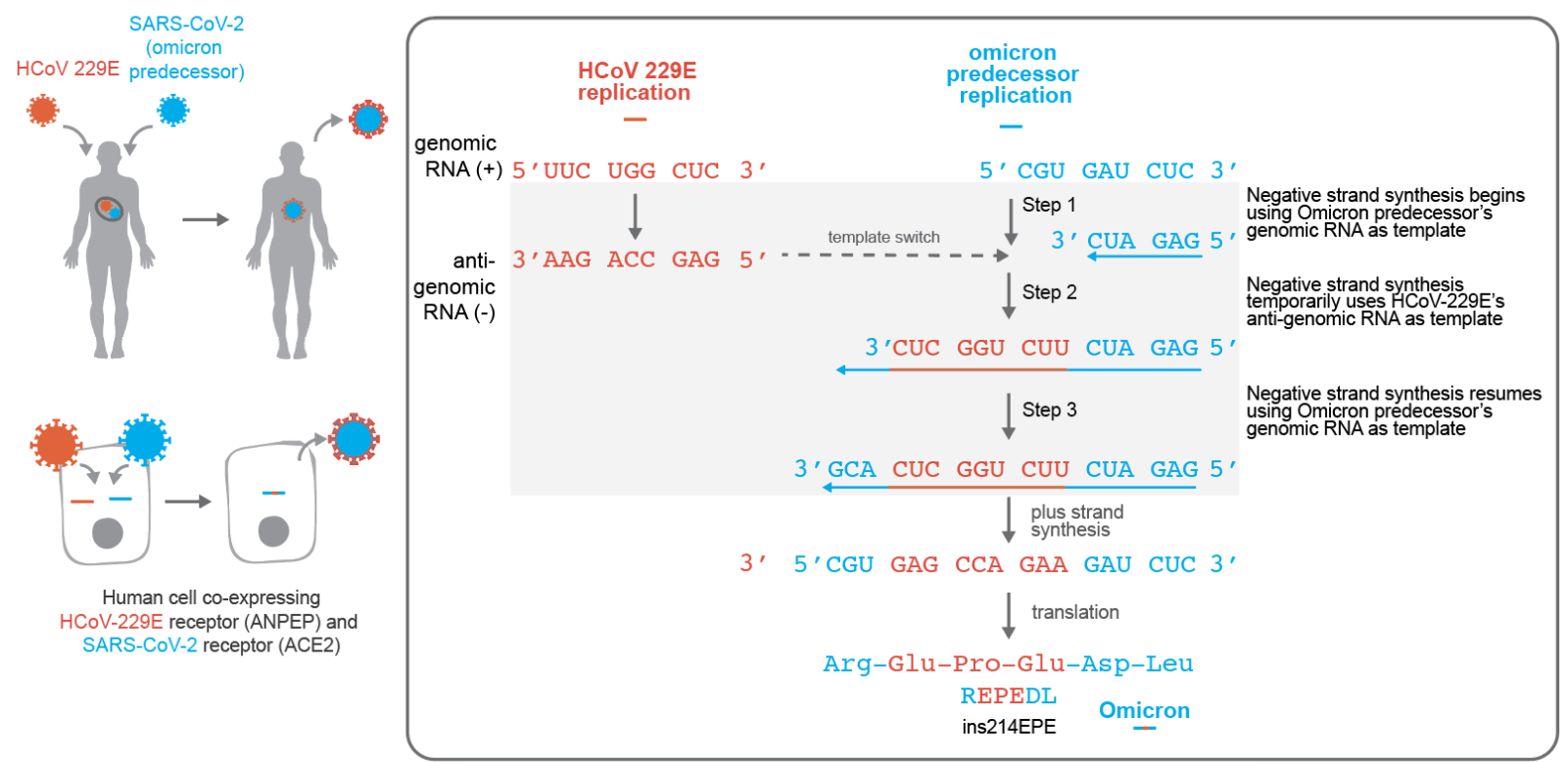

b. Comparison of nucleotides corresponding to the Omicron insert with a homologous match from HCoV 229E

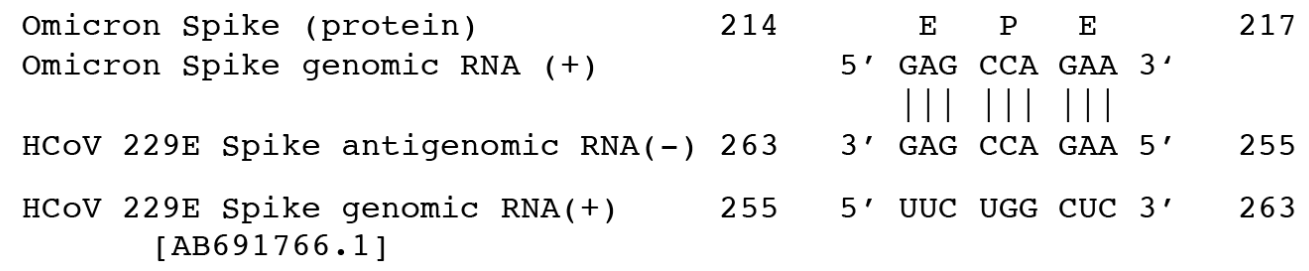

Figure 2. a. Potential mechanism of template switching leading to the generation of the ins214EPE in Omicron. Schematic representations show human body and human cells being infected by Omicron's predecessor variant (blue) and a human coronavirus HCoV-229E (orange). The box shows the potential steps in the template switching involving the genomic RNA (+) of Omicron's predecessor variant and the anti-genomic RNA (-) of HCoV-229E. The steps involving the anti-genomic RNA are shown inside a grey box. b. Comparison of nucleotides corresponding to the Omicron insert with a homologous match from HCoV 229E. Sequence alignment of the genomic regions corresponding to Omicron Spike and HCoV229E Spike are shown. 
Table 1. Comparison of the mutations between the Omicron variant and previously identified variants of interest/concern. The first column denotes the protein domain where the mutation is present. NTD represents the $\mathrm{N}$-terminal domain; RBD represents the receptor binding domain (RBM represents the receptor binding motif); PBCS denotes poly-basic cleavage site and CTD denotes C-terminal domain.

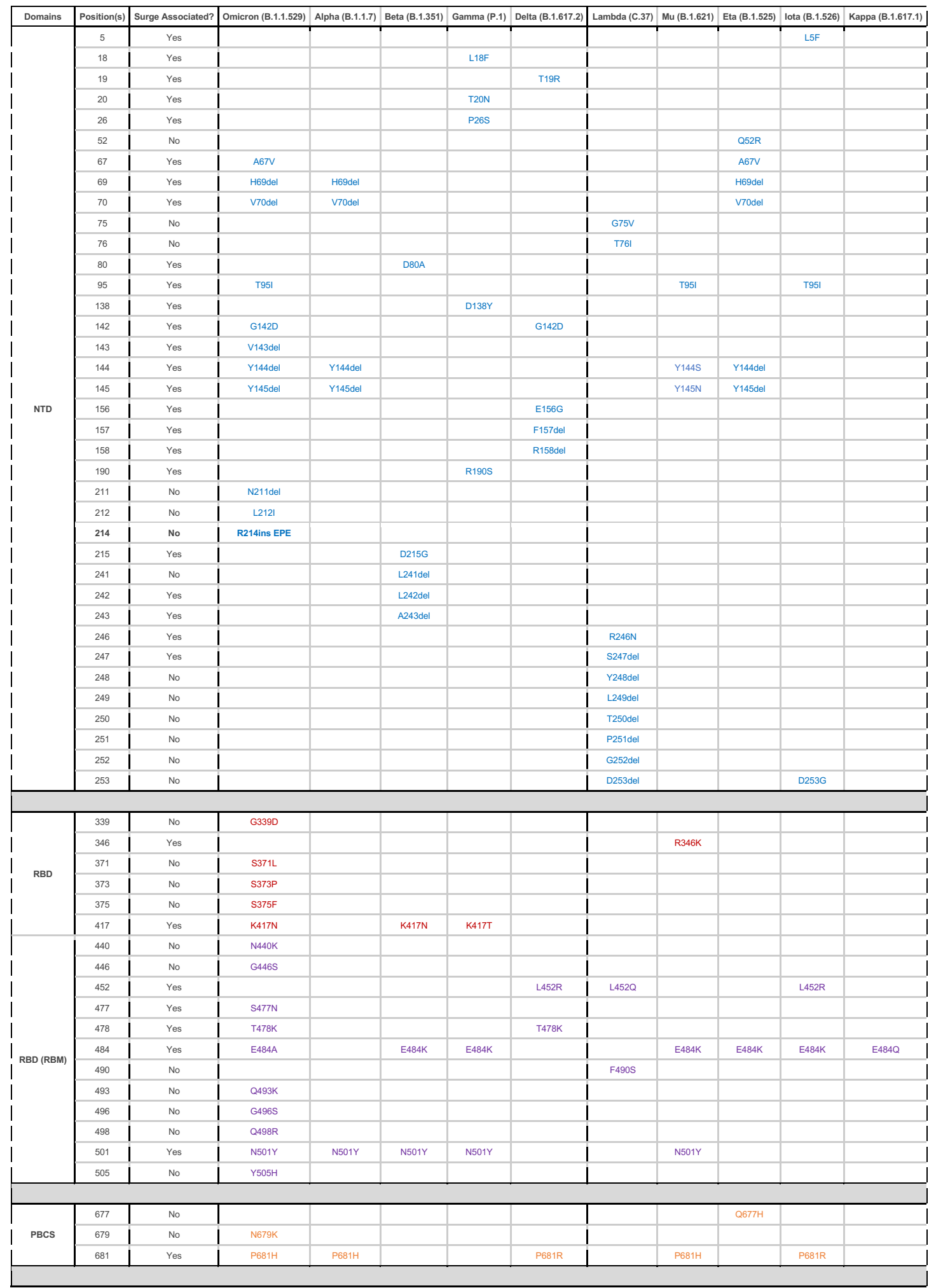




\begin{tabular}{|c|c|c|c|c|c|c|c|c|c|c|c|c|}
\hline \multirow{4}{*}{ SD1/SD2 } & 547 & No & T547K & & & & & & & & & \\
\hline & 570 & Yes & & A570D & & & & & & & & \\
\hline & 614 & Yes & D614G & D614G & D614G & D614G & D614G & D614G & D614G & D614G & D614G & D614G \\
\hline & 655 & Yes & H655Y & & & H655Y & & & & & & \\
\hline & & & & & & & & & & & & \\
\hline \multirow{16}{*}{ Others (CTD) } & 701 & Yes & & & A701V & & & & & & A701V & \\
\hline & 716 & Yes & & T7161 & & & & & & & & \\
\hline & 764 & No & N764K & & & & & & & & & \\
\hline & 796 & No & D796Y & & & & & & & & & \\
\hline & 856 & No & N856K & & & & & & & & & \\
\hline & 859 & Yes & & & & & & T859N & & & & \\
\hline & 888 & No & & & & & & & & F888L & & \\
\hline & 950 & Yes & & & & & D950N & & D950N & & & \\
\hline & 954 & No & Q954H & & & & & & & & & \\
\hline & 969 & No & N969K & & & & & & & & & \\
\hline & 981 & No & L981F & & & & & & & & & \\
\hline & 982 & Yes & & S982A & & & & & & & & \\
\hline & 1027 & Yes & & & & T10271 & & & & & & \\
\hline & 1071 & No & & & & & & & & & & Q1071H \\
\hline & 1118 & Yes & & D1118H & & & & & & & & \\
\hline & 1176 & Yes & & & & V1176F & & & & & & \\
\hline
\end{tabular}




\section{Supplementary Material}

\section{Index:}

Figure S1: Mutational burden of all SARS-CoV-2 proteins for the Omicron variant (B.1.1.529) compared with the Delta variant (B.1.617.2).

Figure S2: Protein sequence alignment of the Spike protein of B.1.1.529 (Omicron) and the reference SARS-CoV-2.

Figure S3: Distribution of prevalence of Omicron proteome mutations.

Figure S4:

Table S1. Coexpression analysis of ACE2 and ANPEP in single cell RNA-sequencing datasets.

Table S2. The number of PANGO lineages that have mutations observed in the Omicron variant's Spike protein. 


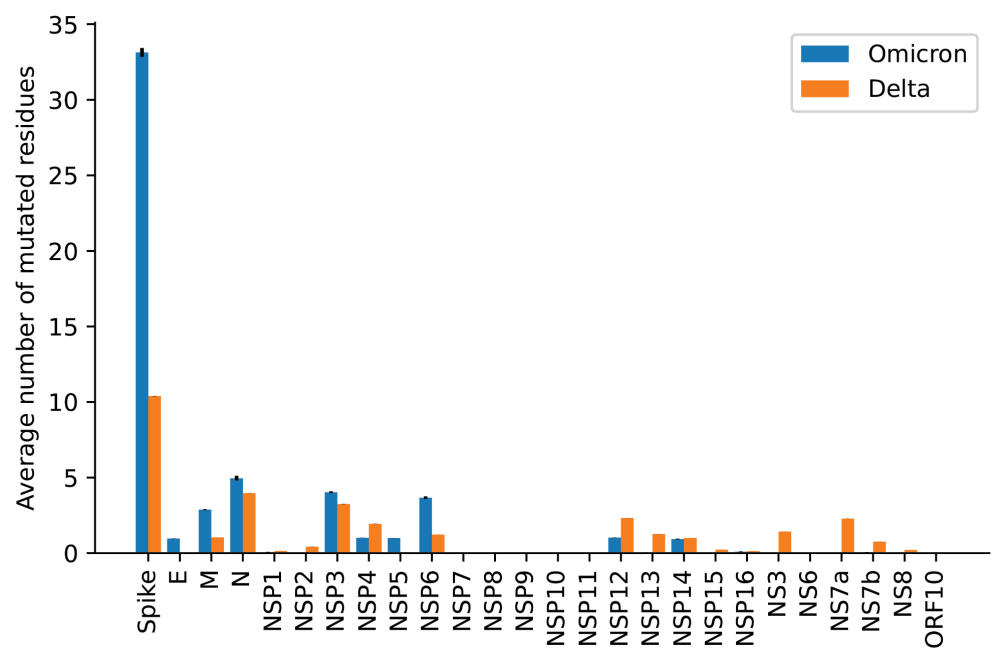

Figure S1: Mutational burden of all SARS-CoV-2 proteins for the Omicron variant (B.1.1.529) compared with the Delta variant (B.1.617.2). A total of 127 Omicron (B.1.1.529) sequences and 169,537 Delta (B.1.617.2) sequences were retrieved from the GISAID on 29 November 2021. Each bar represents the average number of mutations reported for a sequence in each of the SARS-CoV-2 proteins. Error bars represent the standard error of the mean. 


\begin{tabular}{|c|c|c|}
\hline >P0DTC2 & MFVFLVLLPLVSSOCVNLTTRTQLPPAYTNSFTRGVYYPDKVFRSSVLHSTODLFLPFFS & 60 \\
\hline$>$ B. 1.1 .529 & MFVFLVLLPLVSSOCVNLTTRTQLPPAYTNSFTRGVYYPDKVFRSSVLHSTQDLFLPFFS & 60 \\
\hline >P0DTC2 & NVTWFHAIHVSGTNGTKRFDNPVLPFNDGVYFASTEKSNIIRGWIFGTTLDSKTOSLLIV & 120 \\
\hline >B. 1.1 .529 & NVTWFHVI--SGTNGTKRFDNPVLPFNDGVYFASIEKSNIIRGWIFGTTLDSKTQSLLIV & 118 \\
\hline$>$ P0DTC2 & NNATNVVIKVCEFOFCNDPFLGVYYHKNNKSWMESEFRVYSSANNCTFEYVSOPFLMDLE & 180 \\
\hline$>$ B. 1.1 .529 & $\begin{array}{l}\text { NNATNVVIKVCEFOFCNDPFLD---HKNNKSWMESEFRVYSSANNCTFEYVSQPFLMDLE } \\
\text { ins214EPE: }\end{array}$ & 175 \\
\hline >P0DTC2 & GKOGNFKNLREFVFKNIDGYFKIYSKHTPINLVR---DLPQGFSALEPLVDLPIGINITRFOT & 240 \\
\hline >B. 1.1 .529 & GKOGNFKNLREFVFKNIDGYFKIYSKHTPI-IVREPEDLPQGFSALEPLVDLPIGINITRFOT & 237 \\
\hline >P0DTC2 & LLALHRSYLTPGDSSSGWTAGAAAYYVGYLQPRTFLLKYNENGTITDAVDCALDPLSETK & 300 \\
\hline >B. 1.1 .529 & LLALHRSYLTPGDSSSGWTAGAAAYYVGYLQPRTFLLKYNENGTITDAVDCALDPLSETK & 297 \\
\hline$>$ P0DTC2 & CTLKSFTVEKGIYQTSNFRVQPTESIVRFPNITNLCPFGEVFNATRFASVYAWNRKRISN & 360 \\
\hline$>$ B. 1.1 .529 & CTLKSFTVEKGIYQTSNFRVQPTESIVRFPNITNLCPFDEVFNATRFASVYAWNRKRISN & 357 \\
\hline >P0DTC2 & CVADYSVLYNSASFSTFKCYGVSPTKLNDLCFTNVYADSFVIRGDEVRQIAPGQTGKIAD & 420 \\
\hline >B. 1.1 .529 & CVADYSVLYNLAPFFTFKCYGVSPTKLNDLCFTNVYADSFVIRGDEVRQIAPGQTGNIAD & 417 \\
\hline$>$ P0DTC2 & YNYKLPDDFTGCVIAWNSNNLDSKVGGNYNYLYRLFRKSNLKPFERDISTEIYQAGSTPC & 480 \\
\hline$>$ B. 1.1 .529 & YNYKLPDDFTGCVIAWNSNKLDSKVSGNYNYLYRLFRKSNLKPFERDISTEIYQAGNKPC & 477 \\
\hline >P0DTC2 & NGVEGFNCYFPLQSYGFOPTNGVGYQPYRVVVLSFELLHAPATVCGPKKSTNLVKNKCVN & 540 \\
\hline >B. 1.1 .529 & NGVAGFNCYFPLKSYSFRPTYGVVGHQPYRVVVLSFELLHAPATVCGPKKSTNLVKNKCVN & 537 \\
\hline >P0DTC2 & FNFNGLTGTGVLTESNKKFLPFQOFGRDIADTTDAVRDPOTLEILDITPCSFGGVSVITP & 600 \\
\hline$>$ B. 1.1 .529 & FNFNGLKGTGVLTESNKKFLPFOOFGRDIADTTDAVRDPOTLEILDITPCSFGGVSVITP & 597 \\
\hline >P0DTC2 & GTNTSNOVAVLYQDVNCTEVPVAIHADQLTPTWRVYSTGSNVFOTRAGCLIGAEHVNNSY & 660 \\
\hline$>$ B. 1.1 .529 & GTNTSNQVAVLYQGVNCTEVPVAIHADQLTPTWRVYSTGSNVFQTRAGCLIGAEYVNNSY & 657 \\
\hline >P0DTC2 & ECDIPIGAGICASYQTQTNSPRRARSVASOSIIAYTMSLGAENSVAYSNNSIAIPTNFTI & 720 \\
\hline >B. 1.1 .529 & ECDIPIGAGICASYOTOTKSHRRARSVASOSIIAYTMSLGAENSVAYSNNSIAIPTNFTI & 717 \\
\hline >P0DTC2 & SVTTEILPVSMTKTSVDCTMY ICGDSTECSNLLLQYGSFCTQLNRALTGIAVEQDKNTOE & 780 \\
\hline$>$ B. 1.1 .529 & SVTTEILPVSMTKTSVDCTMY ICGDSTECSNLLLQYGSFCTQLKRALTGIAVEQDKNTOE & 777 \\
\hline >P0DTC2 & VFAQVKQIYKTPPIKDFGGFNFSQILPDPSKPSKRSFIEDLLFNKVTLADAGFIKQYGDC & 840 \\
\hline$>$ B. 1.1 .529 & VFAQVKQIYKTPPIKYFGGFNFSQILPDPSKPSKRSF IEDLLFNKVTLADAGFIKQYGDC & 837 \\
\hline >P0DTC2 & LGDIAARDLICAQKFNGLTVLPPLLTDEMIAQYTSALLAGTITSGWTFGAGAALQIPFAM & 900 \\
\hline$>$ B. 1.1 .529 & LGDIAARDLICAQKFKGLTVLPPLLTDEMIAQYTSALLAGTITSGWTFGAGAALQIPFAM & 897 \\
\hline >P0DTC2 & QMAYRFNGIGVTQNVLYENOKLIANOFNSAIGKIQDSLSSTASALGKLQDVVNONAQALN & 960 \\
\hline$>$ B. 1.1 .529 & QMAYRFNGIGVTQNVLYENQKLIANQFNSAIGKIQDSLSSTASALGKLQDVVNHNAQALN & 957 \\
\hline$>$ P0DTC2 & TLVKQLSSNFGAISSVLNDILSRLDKVEAEVQIDRLITGRLQSLQTYVTQQLIRAAEIRA & 1020 \\
\hline$>$ B. 1.1 .529 & TLVKQLSSKFGAISSVLNDIFSRLDKVEAEVQIDRLITGRLQSLQTYVTQQLIRAAEIRA & 1017 \\
\hline >P0DTC2 & SANLAATKMSECVLGQSKRVDFCGKGYHLMSFPQSAPHGVVFLHVTYVPAOEKNFTTAPA & 1080 \\
\hline >B. 1.1 .529 & SANLAATKMSECVLGQSKRVDFCGKGYHLMSFPQSAPHGVVFLHVTYVPAQEKNFTTAPA & 1077 \\
\hline >P0DTC2 & ICHDGKAHFPREGVFVSNGTHWFVTORNFYEPOI ITTDNTFVSGNCDVVIGIVNNTVYDP & 1140 \\
\hline >B. 1.1 .529 & ICHDGKAHFPREGVFVSNGTHWFVTQRNFYEPOI ITTDNTFVSGNCDVVIGIVNNTVYDP & 1137 \\
\hline >PODTC2 & LQPELDSFKEELDKYFKNHTSPDVDLGDISGINASVVNIOKEIDRLNEVAKNLNESLIDL & 1200 \\
\hline$>$ B. 1.1 .529 & LQPELDSFKEELDKYFKNHTSPDVDLGDISGINASVVNIQKEIDRLNEVAKNLNESLIDL & 1197 \\
\hline >P0DTC2 & QELGKYEQY I KWPWY IWLGF I AGLIAIVMVTI IMLCCMTSCCSCLKGCCSCGSCCKFDEDD & 1260 \\
\hline$>$ B. 1.1 .529 & QELGKYEQY I KWPWY IWLGF I AGL I AIVMVT IMLCCMTSCCSCLKGCCSCGSCCKFDEDD & 1257 \\
\hline >P0DTC2 & SEPVLKGVKLHYT & 1273 \\
\hline >B.1.1.529 & SEPVLKGVKLHYT & 1270 \\
\hline
\end{tabular}

Figure S2. Protein sequence alignment of the Spike protein of B.1.1.529 (Omicron) and the reference SARS-CoV-2. The uniprot Accession number of the reference SARS-CoV-2 Spike protein is P0DTC2 (https://www.uniprot.org/uniprot/P0DTC2). The insertion mutation ins214EPE is highlighted in blue. 

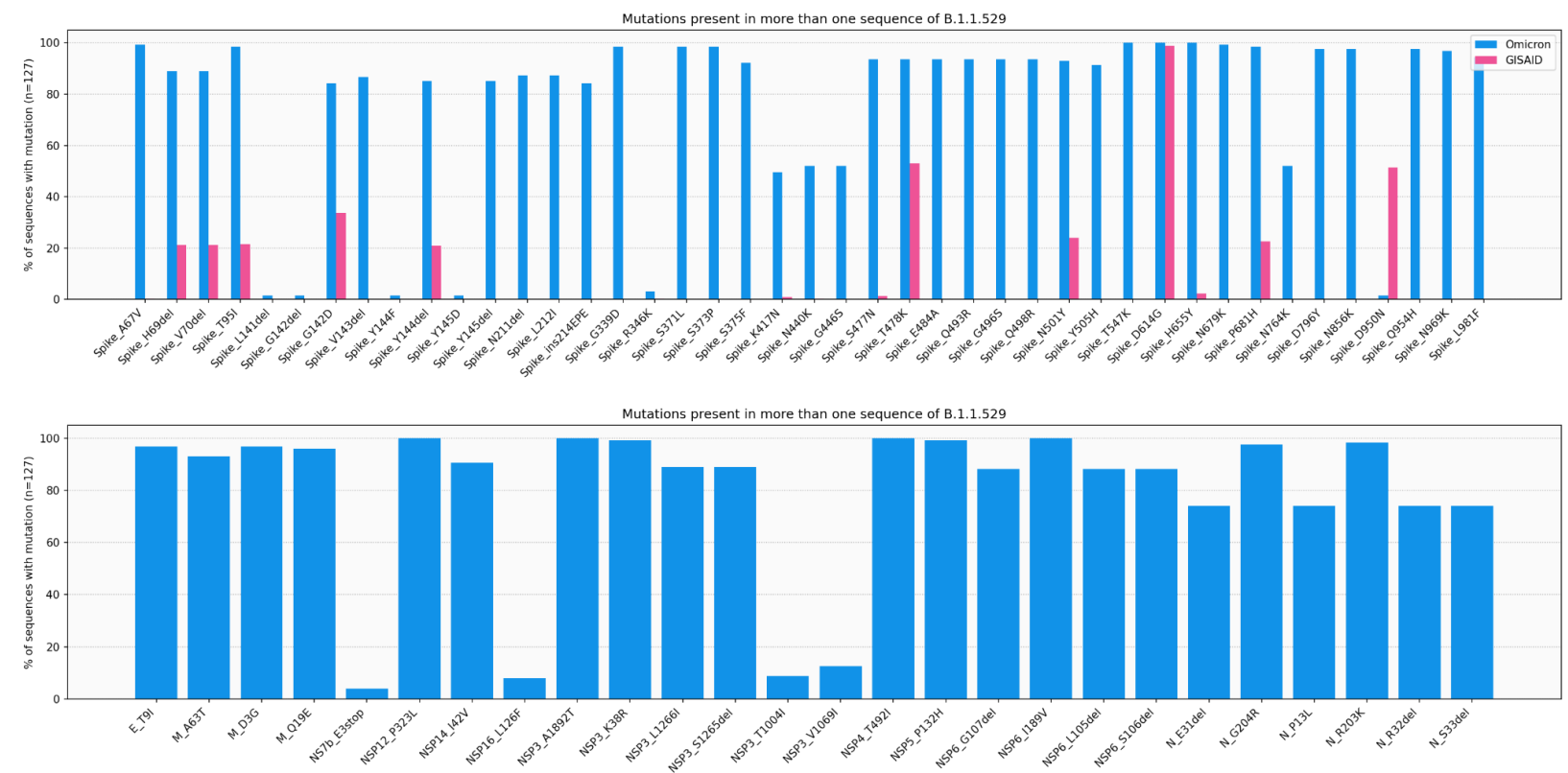

Figure S3: Distribution of prevalence of Omicron proteome mutations. A total of 127 Omicron (B.1.1.529) variant sequences from 8 countries were retrieved from the GISAID on 29 November 2021. Each blue bar represents the percentage of the 127 sequences that contain a given mutation, for the Spike protein (top) or other viral proteins (bottom). As a reference, the red bars represent the percentage of nonOmicron GISAID sequences that contain the exact same Spike-protein mutation. The figure includes only mutations which are present in more than one sequence.

\begin{tabular}{|c|c|c|c|c|c|c|c|c|c|}
\hline & Description & $\begin{array}{c}\text { Scientific } \\
\text { Name } \\
\downarrow\end{array}$ & $\begin{array}{l}\text { Max } \\
\text { Score } \\
\square\end{array}$ & $\begin{array}{l}\text { Total } \\
\text { Score } \\
\square\end{array}$ & $\begin{array}{l}\text { Query } \\
\text { Cover } \\
\end{array}$ & $\underset{\text { value }}{\mathrm{E}}$ & $\begin{array}{l}\text { Per. } \\
\text { Ident } \\
\nabla\end{array}$ & Acc. Len & Accession \\
\hline \multicolumn{9}{|c|}{ Transcripts } & - \\
\hline$\square$ & Homo sapiens solute carrier family 7 member 8 (SLC7A8), transcript variant 4 , mRNA & Homo sapiens & 28.2 & 28.2 & $93 \%$ & 24 & $100.00 \%$ & 2963 & NM_001267037.2 \\
\hline$\square$ & Homo sapiens solute carrier family 7 member 8 (SLC7A8), transcript variant 5 , non-coding RNA & Homo sapiens & 28.2 & 28.2 & $93 \%$ & 24 & $100.00 \%$ & 3077 & $\underline{N R \quad 049767.2}$ \\
\hline$\square$ & Homo sapiens solute carrier family 7 member 8 (SLC7A8), transcript variant 2 , mRNA & Homo sapiens & 28.2 & 28.2 & $93 \%$ & 24 & $100.00 \%$ & 3117 & NM 182728.3 \\
\hline$\square$ & Homo sapiens transmembrane protein 245 (TMEM245), mRNA & Homo sapiens & 28.2 & 28.2 & $93 \%$ & 24 & $100.00 \%$ & 7999 & NM_032012.4 \\
\hline$\square$ & Homo sapiens solute carrier family 7 member 8 (SLC7A8), transcript variant 1 , mRNA & Homo sapiens & 28.2 & 28.2 & $93 \%$ & 24 & $100.00 \%$ & 4236 & NM_012244.4 \\
\hline$\square$ & Homo sapiens apolipoprotein A4 (APOA4), mRNA & Homo sapiens & 28.2 & 28.2 & $93 \%$ & 24 & $100.00 \%$ & 1471 & NM_000482.4 \\
\hline$\square$ & PREDICTED: Homo sapiens transmembrane protein 245 (TMEM245), transcript variant X6, mRNA & Homo sapiens & 28.2 & 28.2 & $93 \%$ & 24 & $100.00 \%$ & 7747 & $\underline{X M \quad 011518452.2}$ \\
\hline$\square$ & PREDICTED: Homo sapiens transmembrane protein 245 (TMEM245), transcript variant X4, mRNA & Homo sapiens & 28.2 & 28.2 & $93 \%$ & 24 & $100.00 \%$ & 7865 & XM_011518449.2 \\
\hline$\square$ & PREDICTED: Homo sapiens uncharacterized LOC101927857 (LOC101927857), transcript variant X2, nC... & Homo sapiens & 28.2 & 28.2 & $93 \%$ & 24 & $100.00 \%$ & 4327 & $\underline{X R \_001753417.2}$ \\
\hline$\square$ & PREDICTED: Homo sapiens transmembrane protein 245 (TMEM245), transcript variant X7, mRNA & Homo sapiens & 28.2 & 28.2 & $93 \%$ & 24 & $100.00 \%$ & 7722 & XM 017014572.1 \\
\hline
\end{tabular}

Figure S4: Identification of nucleotide sequences in the human genome homologous to Omicron insertion ins214EPE using NCBI BLAST. Subset of 763 hits based on a NCBI BLAST search for 'CGTGAGCCAGAAGAT', which includes the two possible insertion nucleotide sequences (GAGCCAGAA, GCCAGAAGA) that can code for Omicron's ins214EPE. The search was performed against the database of human genome plus transcriptome database (Human $\mathrm{G}+\mathrm{T}$ ) containing 160592 sequences. The top hits include SLC7A8 and TMEM245. 
Table S1. Number of PANGO lineages with mutations in the Omicron variant's Spike protein.

\begin{tabular}{|c|c|}
\hline AA Substitutions & Number of Pango lineage in which mutation has observed As of 29 Nov 2021 \\
\hline Spike_A67V & 250 \\
\hline Spike_H69del & 379 \\
\hline Spike_V70del & 378 \\
\hline Spike_T95I & 509 \\
\hline Spike_G142D & 246 \\
\hline Spike_V143del & 294 \\
\hline Spike_Y144del & 573 \\
\hline Spike_Y145del & 149 \\
\hline Spike_N211del & 90 \\
\hline Spike_L212I & 72 \\
\hline Spike_ins214EPE & 0 \\
\hline Spike_G339D & 62 \\
\hline Spike_S371L & 2 \\
\hline Spike_S373P & 63 \\
\hline Spike_S375F & 48 \\
\hline Spike_K417N & 162 \\
\hline Spike_N440K & 82 \\
\hline Spike_G446S & 87 \\
\hline Spike_S477N & 254 \\
\hline Spike_T478K & 304 \\
\hline Spike_E484A & 87 \\
\hline Spike_Q493K & 28 \\
\hline Spike_G496S & 18 \\
\hline Spike_Q498R & 37 \\
\hline Spike_N501Y & 348 \\
\hline Spike_Y505H & 15 \\
\hline Spike_T547K & 76 \\
\hline Spike_D614G & 1464 \\
\hline Spike_H655Y & 357 \\
\hline Spike_N679K & 122 \\
\hline Spike_P681H & 374 \\
\hline Spike_N764K & 32 \\
\hline Spike_D796Y & 192 \\
\hline Spike_N856K & 20 \\
\hline Spike_Q954H & 14 \\
\hline Spike_N969K & 25 \\
\hline Spike_L981F & 15 \\
\hline
\end{tabular}


Table S2. Co-expression analysis of ACE2 and ANPEP in single cell RNA-sequencing datasets. The number and percent of each cell type expressing ACE2, ANPEP, or both ACE2 and ANPEP are shown. The Observed/Expected Ratio is calculated assuming that expression of ACE2 and ANPEP is distributed randomly across the analyzed cells. Specifically, the Observed/Expected Ratio is calculated by dividing the co-expressing percentage by the product of the individual expression percentages and multiplying by 100 . In the first row, "All studies" corresponds to the set of studies that are hosted in the previously described Single Cell application at academia.nferx.com. ${ }^{30,34}$ The analyzed enterocytes are derived from a study of ileal biopsies from Crohn's Disease patients. ${ }^{35}$ The analyzed respiratory ciliated cells and FOXN4+ respiratory epithelial cells are derived from a study of nasopharyngeal and bronchial samples from COVID19 patients and healthy controls. ${ }^{36}$

\begin{tabular}{|c|c|c|c|c|c|c|}
\hline $\begin{array}{c}\text { Study source } \\
\text { (PMID) }\end{array}$ & Cell Type & $\begin{array}{c}\text { Number of } \\
\text { cells }\end{array}$ & $\begin{array}{c}\text { Cells } \\
\text { expressing } \\
\text { ACE2 (\%) }\end{array}$ & $\begin{array}{c}\text { Cells } \\
\text { expressing } \\
\text { ANPEP (\%) }\end{array}$ & $\begin{array}{c}\text { Cells co- } \\
\text { expressing } \\
\text { ACE2 and } \\
\text { ANPEP (\%) }\end{array}$ & $\begin{array}{c}\text { Observed } \\
\text { / Expected } \\
\text { Ratio }\end{array}$ \\
\hline All studies & All cells & $2.8 M$ & $\begin{array}{c}26.9 K \\
(0.96 \%)\end{array}$ & $\begin{array}{c}221.3 K \\
(7.9 \%)\end{array}$ & $\begin{array}{c}13.5 K \\
(0.48 \%)\end{array}$ & 6.4 \\
\hline PMID 31474370 & All cells & $32.5 K$ & $\begin{array}{c}432 \\
(1.3 \%)\end{array}$ & $\begin{array}{c}1.4 K \\
(4.3 \%)\end{array}$ & $\begin{array}{c}403 \\
(1.2 \%)\end{array}$ & 21.7 \\
\cline { 2 - 7 } & Enterocytes & 809 & $\begin{array}{c}337 \\
(41.7 \%)\end{array}$ & $\begin{array}{c}757 \\
(93.6 \%)\end{array}$ & $\begin{array}{c}329 \\
(40.7 \%)\end{array}$ & 1.04 \\
\hline \multirow{3}{*}{ PMID 32591762 } & All cells & $135.6 K$ & $\begin{array}{c}3 K \\
(2.2 \%)\end{array}$ & $\begin{array}{c}15.4 K \\
(11.4 \%)\end{array}$ & $\begin{array}{c}1.5 K \\
(1.1 \%)\end{array}$ & 4.4 \\
\cline { 2 - 7 } & $\begin{array}{c}\text { Respiratory } \\
\text { ciliated cells }\end{array}$ & $5.8 \mathrm{~K}$ & $\begin{array}{c}827 \\
(14.3 \%)\end{array}$ & $\begin{array}{c}1.2 \mathrm{~K} \\
(20.7 \%)\end{array}$ & $\begin{array}{c}217 \\
(3.7 \%)\end{array}$ & 1.3 \\
\cline { 2 - 7 } & $\begin{array}{c}\text { FOXN4+ } \\
\text { respiratory } \\
\text { epithelial cells }\end{array}$ & 787 & $\begin{array}{c}89 \\
(11.3 \%)\end{array}$ & $\begin{array}{c}302 \\
(38.4 \%)\end{array}$ & $\begin{array}{c}56 \\
(7.1 \%)\end{array}$ & 1.6 \\
\hline
\end{tabular}

